\title{
Proposal for the assessment of quantitative dermal exposure limits in occupational environments: part 2. Feasibility study for application in an exposure scenario for MDA by two different dermal exposure sampling methods
}

\author{
D H Brouwer, L Hoogendoorn, P M J Bos, P J Boogaard, J J van Hemmen
}

\begin{abstract}
Objective-To evaluate two different techniques for assessing dermal exposure to $4,4^{\prime}$ - methylene dianiline (MDA) in a field study. The results were used to test the applicability of a recently proposed quantitative dermal occupational exposure limit (DOEL) for MDA in a workplace scenario.

Methods-For two consecutive weeks six workers were monitored for exposure to MDA in a factory that made glass fibre reinforced resin pipes. Dermal exposure of the hands and forearms was assessed during week 1 by a surrogate skin technique (cotton monitoring gloves) and during week 2 by a removal technique (hand wash). As well as the dermal exposure sampling, biological monitoring, measurement of MDA excretion in urine over 24 hours, occurred during week 2 . Surface contamination of the workplace and equipment was monitored qualita-
\end{abstract} tively by colorimetric wipe samples.

Results and conclusions-Geometric means of daily exposure ranged from 81-1762 $\mu \mathrm{g}$ MDA for glove monitoring and from 84-1783 $\mu \mathrm{g}$ MDA for hand washes. No significant differences, except for one worker, were found between exposure of the hands in weeks 1 and 2. Significant differences between the mean daily exposure of the hands (for both weeks and sampling methods) were found for all workers. The results of the colorimetric wipe samples indicated a general contamination of the workplace and equipment. Excretion of MDA in 24 hour urine samples ranged from 8 to $249 \mu \mathrm{g}$ MDA, whereas cumulative MDA excretion over a week ranged from 82 to $717 \mu \mathrm{g}$ MDA. Cumulative hand wash and MDA excretion results over a week showed a high correlation $\left(R^{2}=0.94\right)$. The highest actual daily dermal exposure found seemed to be about $4 \mathrm{mg}$ (hand wash worker $A$ on day 4), about $25 \%$ of the external DOEL. Testing of compliance by means of a biological limit value (BLV) led to similar results for the same worker. It is concluded that both dermal exposure monitoring methods were applicable and showed a compatible performance in the present exposure scenario, where the exposure relevant to der- mal absorption is considered mainly restricted to hands. The concept for a DOEL seemed to be relevant and applicable for compliance testing and health surveillance in the situation under investigation.

(Occup Environ Med 1998;55:805-811)

Keywords: dermal exposure monitoring; MDA; biological monitoring; compliance; dermal occupational exposure limit

In the first part of this article a conceptual procedure to derive a dermal occupational exposure limit (DOEL) has been presented. ${ }^{1}$ For testing whether occupational conditions are in compliance with a DOEL, it is essential that validated monitoring techniques are available. In risk assessment the assessment of whether a particular workplace environment or a given combination of tasks of a worker could give rise to hazardous dermal exposure may be a requirement. As a consequence it may be necessary to monitor the workplace or the workers involved, which requires different approaches.

DERMAL EXPOSURE PROCESSES AND PATHWAYS Contamination of the skin can be considered to be the result of dynamic processes that may be characterised as loading and removing. ${ }^{2}$ The loading process is the result of: $(a)$ deposition of and impaction with aerosols present in the work environment; (b) immersion in a liquid or powder; (c) contact with a contaminated surface. The removal processes leading to decontamination are vaporisation of the contaminant, immersion in a clean liquid, and by mechanical forces (vibrations and shocks). These processes are variable and may change with time and be described as intermittent and continuous.

Fenske defined dermal exposure as the product of skin loading rate (mass/skin surface area/unit time) and area exposure $\left(\mathrm{cm}^{2}\right){ }^{3}$ Dermal exposure is expressed in units of mass per unit time $(\mu \mathrm{g} / \mathrm{h})$. Cherrie and Robertson proposed an alternative definition which recognises the biological process involved in skin absorption - that is, the concentration of the substance at the skin surface. ${ }^{4}$ They argue that measurement of mass of material on the skin surface may be misleading in cases in which the concentration of the material is variable. As measurement of exposure with the suggested 
definition of Cherrie and Robertson is presently not possible, the definition of Fenske has been adopted for the aim of the present study:

$$
D E=\Sigma\left(S L R_{1} \times A_{1}\right)+. .+\left(S L R_{n} \times A_{n}\right),
$$

where $D E=$ dermal exposure $(\mu \mathrm{g} / \mathrm{h})$; $S L R=$ skin loading rate for each region $\left(\mu \mathrm{g} / \mathrm{cm}^{2}\right)$ h); $A=$ exposed skin area for each region $\left(\mathrm{cm}^{2}\right)$; and $n=\mathrm{n}^{\text {th }}$ skin region.

\section{DERMAL EXPOSURE SAMPLING}

In the present report, it had to be ascertained that the sampling techniques met the accuracy requirements of the occupational exposure limits for precision, specificity, and sensitivity. The following requirements have been specified for an ideal assessment method for dermal exposure ${ }^{25}$ : (a) measure the amount that is available for penetration through the skin; $(b)$ estimate skin loading unbiased by duration and time of sampling; (c) enable repeated sampling in time; $(d)$ is applicable for all relevant anatomical regions; (e) mimics the various processes of loading and removing appropriately; and $(f)$ have high resolution, low limits of detection, and is sufficiently validated.

DERMAL EXPOSURE ASSESSMENT TECHNIQUES

Skin exposure sampling techniques fall into three categories: surrogate skin-for example, monitoring gloves, patches; chemical removal-for example, washing and rinsing, skin wipes; and tracer techniques - for example, fluorescent tracer in combination with image processing techniques. These categories have been described extensively by Fenske ${ }^{3}$ and discussed with respect to the requirements given before by Van Hemmen and Brouwer. ${ }^{2}$

It was concluded that none of the sampling techniques considered meet all requirements for an ideal sampling technique. For the application of such techniques for testing compliance of occupational dermal exposure with a DOEL, both the surrogate skin and the removal sampling techniques are limited by their poor resolution properties. The dermal dose/unit area $\left(D_{A}\right)$, the mass loading of the skin/unit of surface area, can only be expressed as the average $D_{A}$ over the (skin) area which has been sampled. However, we worked from a maximal (cumulative) skin loading. In the most conservative approach gross skin loading resulting from a dermal exposure process or cumulative mass estimation of all exposed body regions, should be added to ascertain compliance with a DOEL. Because of the ease of use for surrogate skin and removal techniques, dermal exposure was assessed by both these techniques in the present study.

STUDY OBJECTIVE

The objective of the study was to investigate the feasibility of the application of dermal exposure sampling techniques for testing compliance of occupational dermal exposure with a conceptual DOEL in a field study. A plant producing glass fibre reinforced resin pipes offered suitable conditions for this. Workers were exposed to 4,4'- methylene dianiline (MDA) only through the skin; 8 hour time weighted average (TWA) concentrations (by personal air sampling) were $<3 \mathrm{ppb}$. Also a suitable biological monitoring method is available for MDA. ${ }^{6}$ These findings suggest that exposure to MDA may only have occurred by skin contact. Hence, the exposure scenario for MDA offers the possible study of the applicability of a DOEL in an occupational work environment by comparing external exposure measurements with an external DOEL and internal exposure measurements (biological monitoring) with a biological limit value (internal DOEL)). Two different techniques were used to assess external exposure - that is, a surrogate skin and a removal technique. In part I a DOEL for MDA has been derived. ${ }^{1}$

\section{Material and methods}

PROCESS AND EXPOSURE

In the production of reinforced plastic pipes glass fibre sheets are lined and then led, on a mould, through a bath of resin and hardener. The bath contains a $(106: 20 \mathrm{w} / \mathrm{w})$ mixture of an Epikote 826 solution in xylene (100:6 w/w) and MDA diluted with triethyleneamine $(27: 13 \mathrm{w} / \mathrm{w})$, so the MDA content is about $12 \%$. The air is removed from the resin by pressing the sheet with a brush or a roller on to the mould. The temperature of the mould is about $60^{\circ} \mathrm{C}$. After a period of about 20 minutes in a furnace at $70^{\circ} \mathrm{C}$, subsequent winding of glass fibre filaments on to the mould occurs. The filaments are also led through a bath of resin and hardener at $65^{\circ} \mathrm{C}$ before winding. The MDA content of this bath is about $21 \%$. The bath is placed on a trolly that runs down the length of the mould. Baths are filled manually with buckets that are filled at the so called day station (tempory container). Excessive resin is removed from the mould during winding with a flexible spatula or a bucket while pressing the glass fibre to the mould. The thickness of the pipe is checked by a marking gauge or a measuring tape. A label is put on to the pipe, before the last layer of resin and glass fibre. Then the moulds are removed and the pipes are transported to a furnace at 100$125^{\circ} \mathrm{C}$ for further hardening.

Most tasks are performed with protective gloves (coating of natural rubber on cotton) and a non-woven disposable coverall (Tyvek). Activities performed with bare hands that may result in potential dermal exposure are removal of excessive resin, incidental leading the glass fibre filaments through parts of the machine, measuring large diameter pipes with a measuring tape, and the use of scissors and labelling the pipes. Also, exposure may occur during the removal of the protective gloves, due to cross contamination of surfaces-for example, machine buttons-and due to splashes during lining and winding. Extensive air monitoring programmes for the previous 3 years showed no detectable inhalation exposure (data not presented).

STUDY POPULATION AND SAMPLING STRATEGY Six men-two liners and four windersparticipated in the 2 week study. The first week 
during the afternoon shift (1400-2200), and the second week during the morning shift (0600-1400). During the first week dermal exposure of the hands of three out of six workers was assessed by a hand washing method, at the start of a break, and immediately after the shift. Urine was collected for 24 hours from the start of a workshift until the start of the next shift for five consecutive work days and during the weekend afterwards. Worker F had a six day working week. Before the first day of monitoring a spot sample of urine was delivered.

Dermal exposure of the hands of the other three workers was monitored by cotton glove monitors which were worn beneath the protective gloves. During the second week dermal exposure of the first three workers was assessed by cotton glove monitors, whereas the other three workers entered the hand wash biological monitoring programme. To ensure similar exposure scenarios workers did not change machines or major activities-lining or winding - during the monitoring period.

Dermal exposure of other parts of the body was assessed by visual inspection of the coverall and wipe sampling of the inside of the coverall and the skin in case of splashes. Also, surface wipes were performed on workplace surfaces.

\section{ASSESSMENT OF HAND EXPOSURE}

Hand wash method

Workers were asked to wash their hands twice with about $1.5 \mathrm{ml}$ of a hypoallergic soap (Sporex, Kimberly-Clark, Veenendaal, The Netherlands) for 15 seconds then to rinse them with tap water. A specially designed hand washing device was used, consisting of a tube attached to the tap of the water supply in the changing room, an adjustable flow control set at a flow rate of about $1.5 \mathrm{l} / \mathrm{min}$, a tap, a funnel, and a 51 polyethylene bottle to collect the rinse water. After the hand washing the funnel was rinsed and the total volume of the water was assessed by weighing. Immediately after weighing, $50 \mathrm{ml}$ rinse water was transferred by a 50 $\mathrm{ml}$ jug into a $100 \mathrm{ml}$ polyethylene bottle. Ten $\mathrm{ml}$ of hydrochloric acid (37\%) was added to the rinsing samples and stored at room temperature. Within 24 hours the samples were transported to the laboratory and stored at $4^{\circ} \mathrm{C}$ until analysis.

\section{Cotton glove monitoring}

At the start of the shift and after each break prewashed long sleeved cotton gloves (cotton stretch $200 \mathrm{~g} / \mathrm{m}^{2}$, Van der Wee, Riel, The Netherlands) covering about $1300 \mathrm{~cm}^{2}$ were provided to the workers. At the start of the break and after the shift the gloves were removed by the investigators and each pair was transferred into a 11 bottle containing $500 \mathrm{ml}$ of a $0.1 \mathrm{~N}$ potassium hydroxide solution in methanol and stored at room temperature. Within 24 hours the samples were transported to the laboratory and stored at $4^{\circ} \mathrm{C}$ until analysis. Validation experiments in the laboratory showed a decrease of MDA recovery in MDA/resin mixture on the glove of about $30 \%$ within 2 hours when the glove was stored at $30^{\circ} \mathrm{C} .{ }^{7}$ No effect on
MDA recovery was found under the conditions of storage.

Field blanks of gloves and hand wash solutions were taken to determine possible contamination during the sampling procedure.

ASSESSMENT OF BODY EXPOSURE AND SURFACE CONTAMINATION

Contamination of the coveralls was found during and at the end of the shift and the positions of the contamination were registered. After the shift the coverall was removed and turned inside out. At positions on the inside of the coverall where contamination was found surface wipe samples were taken with colorometric surface swypes, according to the manufacturers' procedure (CLI Laboratories, Des Plaines, IL, USA). This is a semiquantitative indicator; the colour indication is sensitive with a dection limit of about 3-5 $\mu \mathrm{g}$ of MDA. ${ }^{8}$ Similar procedures were followed during the sampling of contamined surfaces, operation buttons, equipment, and latches of doors. Surface swypes were contaminated with MDA and MDA/Epikote 828 mixture $(100: 28 \mathrm{w} / \mathrm{w})$ to check their specificity. A colour indication was found, whereas contamination with Epikote 828, triehtylenetriamine, and xylene did not show a colour change. Positions on the skin of the worker similar to contaminated positions of the coverall were wiped with skin swypes (CLI Laboratories, Des Plaines, IL, USA). All workers wore jeans underneath a non-woven disposable coverall (Tyvek). Also, workers A, $\mathrm{G}$, and I wore long sleeved shirts, whereas workers $\mathrm{F}$ and $\mathrm{H}$ wore $\mathrm{T}$-shirts. The torso of worker $\mathrm{E}$ was not covered by work clothing.

BIOLOGICAL MONITORING

Workers collected urine samples for 7 days in portions of 24 hours in 2.51 jerry cans. Spot samples were taken before the start of the first shift and before and after the 7 day period of biological monitoring. After delivery of the samples the volume of the urine samples was measured. From each 24 hour urine sample $20 \mathrm{ml}$ was transferred into a $100 \mathrm{ml}$ polyethylene bottle and $10 \mathrm{ml}$ of hydrochloric acid $(37 \%)$ was added and stored at room temperature. Within 24 hours the samples were tranported to the laboratory and stored at $4^{\circ} \mathrm{C}$ until analysis.

EXTRACTION AND CHEMICAL ANALYSES

By addition of potassium hydroxide the $\mathrm{pH}$ of the rinse samples was adjusted to 12 . After the addition of 4,4'-diaminodiphenylethane (EDA) as an internal standard hexane was used to for extraction. After evaporation of the hexane at $50^{\circ} \mathrm{C}$ the residue was added to the mobile phase (methanol and Pic A (100:95) and injected to an high performance liquid chromatography (HPLC) system (SpH 125 FIX) and detected by ECD (LDC, Milton Roy, USA).

A part of the extraction solution of the cotton glove samples was transferred into a tube and inserted into the mobile phase, including the internal standard EDA. The detection method of MDA was similar to that for the hand rinse samples. The limit of 
Table 1 Results of daily exposure assessment of the hands

\begin{tabular}{|c|c|c|c|c|c|c|c|c|c|c|c|c|c|c|c|}
\hline \multirow[b]{2}{*}{ Worker } & \multirow[b]{2}{*}{ fob title } & \multirow[b]{2}{*}{$\begin{array}{l}\text { Work } \\
\text { station }\end{array}$} & \multicolumn{6}{|c|}{ Hand wash results ( $\mu g \mathrm{MDA})$} & \multicolumn{7}{|c|}{ Glove results ( $\mu g \mathrm{MDA}$ ) } \\
\hline & & & $\underset{(n)}{\text { Samples }}$ & $\begin{array}{l}\text { Samples } \\
<L O Q(n)\end{array}$ & Ranget $\neq$ & $A M \ddagger S D$ & $G M \ddagger$ & $95 \% C I$ & $\begin{array}{l}\text { Samples } \\
(n)\end{array}$ & $\begin{array}{l}\text { Samples } \\
<L O Q(n)\end{array}$ & Ranget $\neq$ & $A M$ & $S D$ & $G M \neq$ & $95 \% C I$ \\
\hline A & Winder & Machine 7 & 19 & 0.00 & $213-4046$ & 20401390 & $1762^{\star}$ & 1717 to 4332 & 20 & 0.00 & $235-1108$ & 656 & 362 & $568^{\star}$ & * 262 to 1233 \\
\hline $\mathrm{F}$ & Winder & Machine 7 & 23 & 0.00 & 995-1875 & $1582 \quad 261$ & 1562 & 1310 to 1862 & 19 & 0.00 & $869-3328$ & 1986 & 1088 & 1783 & 842 to 3772 \\
\hline E & Winder & Machine 3 & 21 & 0.00 & $777-1540$ & 1119290 & 1089 & 789 to 1503 & 19 & 1 & $865-1240$ & 1323 & 375 & 1324 & 924 to 1899 \\
\hline $\mathrm{H}$ & Winder & Machine 3 & 20 & 0.00 & $240-927$ & 397 & 339 & 167 to 688 & 19 & 2 & $188-438$ & 313 & 104 & 316 & 226 to 443 \\
\hline G & Liner & Machine 3 & 20 & 11 & $<39.5-203$ & 92 & 81 & 41 to 160 & 19 & 14 & $<20-925$ & 285 & 396 & 84 & 8 to 862 \\
\hline I & Liner & Machine 7 & 20 & 0.00 & 288-950 & 260 & 496 & 278 to 886 & 18 & 1 & $70-559$ & 264 & 185 & 237 & 98 to 574 \\
\hline
\end{tabular}

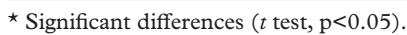

† Range of overall day exposure.

$\ddagger<$ LOQ samples included as $1 / 2$ LOQ.

$\mathrm{AM}=$ arithmetic mean; $\mathrm{GM}=$ geometric mean.

measurement was $10 \mu \mathrm{g} / 1$ for hand rinse samples and $10 \mu \mathrm{g} /$ pair of cotton gloves. The stability of MDA on cotton gloves, on gloves in the presence of Epikote 828, and the recovery of MDA from a soap and water solution were acceptable and are described elsewhere. ${ }^{7}$

Analyses of urine samples have been described extensively elsewhere. ${ }^{9-11}$ Briefly, after addition of the internal standard (EDA) to the urine samples, $\mathrm{N}$-acetylated metabolites were hydrolysed by heating and the addition of potassium hydroxide and extracted by diethyl ether. With hydrochloric acid, free MDA is extracted again from the organic phase, and by diethyl ether, after adjusting to $\mathrm{pH} \mathrm{13,} \mathrm{from} \mathrm{the}$ water phase. Evaporation of the organic phase was performed by a stream of nitrogen at $30^{\circ} \mathrm{C}$. The residue was inserted in the mobile phase and MDA was detected as described previously.

The limit of measurement was $2 \mu \mathrm{g} / 1$ urine and the coefficient of variation was $<10 \%$ for a range of $20-50 \mu \mathrm{g} / 1$ urine. Completeness of 24 hour urine collection was checked by measurement of creatinine concentration in all urine samples.

STATISTICAL ANALYSES AND CALCULATIONS The data were statistically analysed with SOLO statistical system (BMDP Statistical Software, Los Angeles, USA) for personal computers. Differences between average MDA excretion between liners and winders were studied with the Mann-Whitney $U$ test. Hand wash and glove monitor data were log transformed, and geometric means (GMs) and 95\% confidence intervals (95\% CIs) were calculated. Analyses of variance (ANOVAs) were performed on the mean exposure data of the hands for all workers to study differences between exposure assessment methods, whereas $t$ tests were used to study differences in mean daily exposure between the two weeks studied for each worker. For all analyses a probability of $p<0.05$ was considered significant. For data below the limit of detection the half of this limit was used in analyses. This is an acceptable method for handling non-detectable values when the distribution is log normal and the number of detectable values is low. ${ }^{12}$ The relation between the cumulative amount of MDA excreted and the cumulative hand exposure was studied by means of regression analysis.

\section{Results}

ACTUAL EXPOSURE OF THE HANDS

The results of the exposure assessment during the 2 weeks of steady work are given in table 1 . Worker $\mathrm{F}$ worked 6 days during the week in which the hand wash method was used.

Average daily hand wash and glove monitoring results $(\mathrm{GM})$ ranged from 81 to $1762 \mu \mathrm{g}$ MDA, and from 84 to $1783 \mu \mathrm{g}$ MDA, respectively. For risk assessment purposes the average daily exposure is based on the arithmetic means and can be expressed as dermal dose/unit area $\left(D_{A}\right)$. Assuming a surface (skin) area of the skin that was monitored with gloves or by hand washing of about $1300 \mathrm{~cm}^{2}$, the ranges of contamination were 0.07 to 1.56 $\mu \mathrm{g} / \mathrm{cm}^{2}$ and 0.20 to $1.53 \mu \mathrm{g} / \mathrm{cm}^{2}$, respectively. The winders (workers A, F, and E) showed the highest exposure during both weeks, whereas the lowest exposure was found for liner G. As well as his work as a liner, this worker performed other activities not related to MDA during a substantial part of his shift. For this worker about $55 \%$ of both hand wash and glove monitor samples were below the limit of measurement. $t$ Tests showed significant differences of hand exposure in week 1 (hand wash method) and week 2 (glove method) for worker A only. Analyses of variance showed significant differences between the mean daily exposure of the hands (for both weeks and sampling methods) for each worker $(p<0.05)$. Cumulative MDA exposure over the week ranged from 385 $\mu \mathrm{g}$ MDA (worker G) to $10202 \mu \mathrm{g}$ MDA (worker A) and from 430 (worker G) to 9932 $\mu \mathrm{g}$ MDA (worker F) assessed by hand wash and glove monitoring, respectively. During the week in which hand washing was performed worker $\mathrm{F}$ worked for 6 days instead of 5 .

BODY EXPOSURE AND SURFACE CONTAMINATION All workers were inspected once or twice before each break. Visual contamination of the outside of the coverall was found 15 times; most spots were found on the lower legs (front and back) and forearms of the coverall. In nine cases surface swypes showed a colour change. The remaining six spots were due to small splashes. Wiping the skin corresponding to the positions of visual contamination at the coverall by skin swypes did not show exposure.

All surface swypes of the buttons at machine 7 (workers $\mathrm{A}$ and $\mathrm{F}, \mathrm{n}=7$ ) and all but two buttons at machine 3 (workers $\mathrm{E}$ and $\mathrm{H}, \mathrm{n}=7$ ) showed a colour reaction. Six out of 20 swype 
Table 2 Results of biological monitoring

\begin{tabular}{|c|c|c|c|c|c|c|c|c|c|c|c|c|c|}
\hline \multirow[b]{3}{*}{ Day } & \multicolumn{12}{|c|}{$M D A$ excretion in urine } & \multirow[b]{3}{*}{ Remarks } \\
\hline & \multicolumn{2}{|c|}{ Worker $A^{\star}$} & \multicolumn{2}{|c|}{ Worker $E^{\star}$} & \multicolumn{2}{|c|}{ Worker $F^{\star}$} & \multicolumn{2}{|c|}{ Worker $G^{\star}$} & \multicolumn{2}{|c|}{ Worker $H^{\star}$} & \multicolumn{2}{|c|}{ Worker $I^{\star}$} & \\
\hline & $\mu g$ & $\begin{array}{l}\mu g / g \\
\text { creatine }\end{array}$ & $\mu g$ & $\begin{array}{l}\mu g / g \\
\text { creatine }\end{array}$ & $\mu g$ & $\begin{array}{l}\mu g / g \\
\text { creatine }\end{array}$ & $\mu g$ & $\begin{array}{l}\mu g / g \\
\text { creatine }\end{array}$ & $\mu g$ & $\begin{array}{l}\mu g / g \\
\text { creatine }\end{array}$ & $\mu g$ & $\begin{array}{l}\mu g / g \\
\text { creatine }\end{array}$ & \\
\hline 1 & 151 & $\ddagger$ & 5 & 4 & 6 & 3 & 6 & 3 & 14 & 4 & 4 & 2 & Spot sample after 2 days off $\dagger$ \\
\hline 1 & 14 & 9 & 65 & 68 & 103 & 52 & 10 & 5 & 17 & 10 & 18 & 11 & $24 \mathrm{~h}$ urine from start day 1 \\
\hline 2 & 22 & 15 & 115 & 97 & 122 & 65 & 12 & 5 & 29 & 16 & 28 & 18 & $24 \mathrm{~h}$ urine from start day 2 \\
\hline 3 & 69 & 39 & 134 & 99 & 104 & 53 & 8 & 3 & 45 & 28 & 40 & 25 & $24 \mathrm{~h}$ urine from start day 3 \\
\hline 4 & 178 & 102 & 69 & 56 & 73 & 36 & 12 & 4 & 61 & 36 & 34 & 18 & $24 \mathrm{~h}$ urine from start day 4 \\
\hline 5 & 249 & 128 & 133 & 57 & 66 & 35 & 11 & 6 & 50 & 27 & 55 & 29 & $24 \mathrm{~h}$ urine from start day 5 \\
\hline 6 & NA & NA & NA & NA & 66 & 45 & NA & NA & NA & NA & NA & NA & $24 \mathrm{~h}$ urine from start day 6 \\
\hline+1 & 125 & 54 & 9 & 20 & 63 & 44 & 21 & 9 & 61 & 45 & 46 & 21 & $24 \mathrm{~h}$ urine from start first day off \\
\hline+2 & 60 & 38 & 15 & 11 & 27 & 16 & 8 & 7 & 7 & 6 & 26 & 13 & $24 \mathrm{~h}$ urine from start second day off \\
\hline+3 & 411 & 24 & 7 & 5 & 10 & 9 & 5 & 2 & 9 & 2 & 5 & 4 & spot sample after 2 days off $†$ \\
\hline
\end{tabular}

${ }^{\star} \mathrm{p}<0.05$ Mann-Whitney, $v$ group of winders $(\mathrm{A}, \mathrm{F}, \mathrm{E})$ and $v$ liners $(\mathrm{G}, \mathrm{I})$

†MDA excretion expressed in $\mu \mathrm{g} / 1$.

$\ddagger$ Biologically non-representative sample.

$\mathrm{NA}=$ not appropiate

samples indicated a contamination of the buttons of liner unit 3 (worker G), whereas five out of 11 buttons of liner unit 7 (worker I) were contaminated. The handles of all scissors present in the workplace were contaminated. The results of swype tests from other surfaces indicated a general contamination of the workplace,-for example, door handles and doors of cupboards (including the cupboard with protective clothing).

BIOLOGICAL MONITORING

Table 2 summarises the results of the biological monitoring. In all urine spot samples taken before both working weeks MDA was detected, indicating a non- completed excretion of MDA during the period of non-exposure (minimal 56 hours for each worker). For worker A, who was the worker with the highest amount of MDA excreted, the MDA concentration in urine differed by more then a factor of two between the two samples. Figure 1 depicts the excretion pattern of MDA for worker A. Mean amount of MDA excreted in 24 hour urine samples ranged from 8 to $249 \mu \mathrm{g} \mathrm{MDA}$ and differed significantly between the liners $(n=2)$ and the winders $(n=4)$.

RELATION BETWEEN URINARY MDA EXCRETION AND HAND WASH RESULTS

Cumulative MDA excretion over the week ranged from $81 \mu \mathrm{g}$ MDA (worker $\mathrm{G}$ ) to $717 \mu \mathrm{g}$ MDA (worker A). A significant correlation between cumulative MDA excretion and hand wash results over the week was found

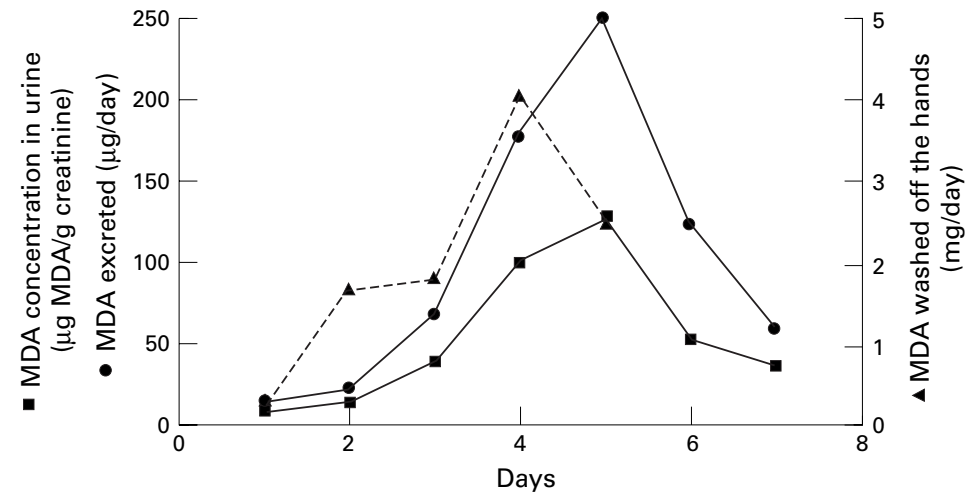

Figure 1 Amount of MDA washed from and excreted by worker $A$ during week 2. (fig 2). Linear regression analyses showed a relation of $\mathrm{MDA}_{\mathrm{URINE}}=115.7+0.058 \mathrm{MDA}_{\mathrm{HAND}} \mathrm{WASH}$ $\left(R^{2}=0.94\right)$.

\section{Discussion and conclusions}

The present study aimed to investigate the feasibility of a quantitative DOEL in an occupational setting. ${ }^{1}$ The feasibility of testing that dermal exposure conditions are in compliance with a DOEL is strongly related to the performance of the dermal exposure sampling technique. Both a hand wash (removal) and a glove monitoring technique (surrogate skin) were used; however, validation of both methods is limited.

Generally, surrogate skin monitoring techniques are considered to overestimate actual skin exposure, as the retention of the cotton fabrics compared with retention of the skin is high. ${ }^{2}$ Average sampling time in the field study was about 80 minutes but never exceeded 120 minutes. Laboratory tests at the temperature conditions underneath the protective glove (about $30^{\circ} \mathrm{C}$ ) showed a decrease of recovery of MDA in the resin mixture from the monitoring glove of about $30 \%$ within two hours. ${ }^{7}$ Therefore, in the present study a slight underestimation of MDA exposure may have occurred. The removal efficiency of MDA from the skin by hand washing has not been investigated. Results of tests of efficiency of removal of pesticides by hand washing performed by volunteers in our institute

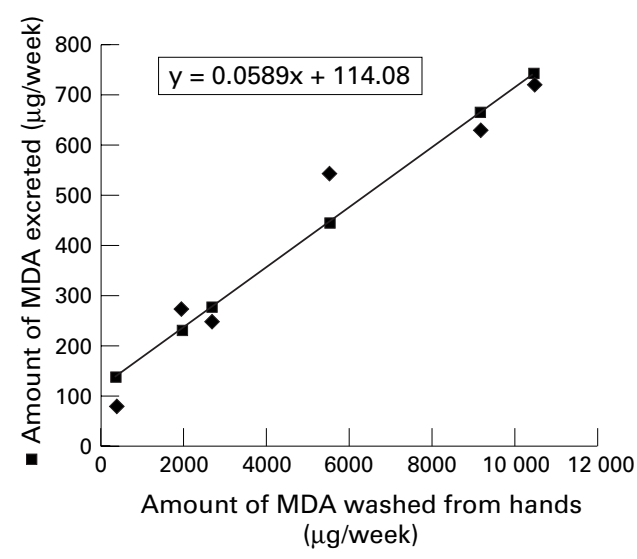

Figure 2 Relation between the cumulative amount of $M D A$ excreted and the cumulative amount of $M D A$ washed off from the hands in 1 week of monitoring. 
after dermal application ranged from about $40 \%$ to $95 \%$. Therefore, a slight underestimation of MDA exposure may occur. Concurrent MDA exposure monitoring by hand washing and biological monitoring is considered applicable for MDA. From data reported by Hewitt et $a l,{ }^{13}$ it can be concluded that washing the hands with liquid soap and water 30 minutes after contamination has occurred will have little effect on removing MDA, as a cutaneous reservoir will already have been established. Therefore, it is indicated that the hand wash method to determine MDA exposure will not interfere with the process of absorption.

The design of the study, however, enables comparison within workers of both methods, assuming a limited variance of exposure due to work activities. The results for hand exposure show large variances within all workers between the days. Despite this finding, for all but one worker no significant differences were found between both weeks of exposure, indicating a compatible performance of both methods of exposure assessment.

Quantitative assessment of exposure was limited to hand exposure, assuming no substantial exposure of other parts of the skin. The exposure of the hands is likely to result from the performance of tasks without protective gloves and the cross contamination of handles, equipment, and buttons, and seems to be more structural, whereas exposure of other parts of the body-for example, resulting from splashes - seems to be more incidental exposure and not to contribute substantially to the total external exposure. This is supported by the results from the swype tests performed at positions of the body underneath locations where splashes were found visually, although the relatively high limit of detection (about 3-5 $\mu \mathrm{g}$ ) does not entirely exclude a minor exposure of other body parts. The surface swypes seem to be useful as part of a workplace contamination control programme to prevent exposure due to contact with contaminated surfaces.

As $62 \%$ of the total excreted dose of ${ }^{14} \mathrm{C}$ labelled MDA after dermal administration to rhesus monkeys was excreted in the first 24 hours, ${ }^{9}$ comparison of MDA from hand washes on a daily basis and the corresponding MDA in 24 hour urine samples was considered inappropriate. Therefore, results of cumulative hand exposure and cumulative MDA excreted in urine over 1 week (including about 2 days of non-exposure) were used for comparison. The strong association between the cumulative amount of MDA excreted in urine and washed off, the absence of detectable contamination at other body parts, and the assumption of negligible inhalation exposure, based on a previous study $^{6}$ suggest that the exposure relevant for dermal exposure is mainly restricted to the hands.

As documented in part 1 for MDA the DOEL associated with an excess incidence of mortality of $4 / 1000$, interpreted as $D_{A} \times A$, was set at $16 \mathrm{mg} /$ day. ${ }^{1}$ In the present study the highest actual daily dermal exposure found seemed to be about $4 \mathrm{mg}$ (hand wash worker A on day 4 ), about $25 \%$ of the external DOEL. Testing of compliance by means of a biological limit value (BLV) led to similar results. A BLV of $1280 \mu \mathrm{g}$ MDA in 24 hour urine samples can be derived from the DOEL of $16 \mathrm{mg} /$ day, assuming a urinary excretion of $8 \%$ of the absorbed amount of MDA. The highest excreted amount of MDA for worker A ( $249 \mu \mathrm{g}$ MDA on day 5) was about $20 \%$ of the BLV. This agreement confirms the applicability of a DOEL as an instrument for surveillance. However, the fact that MDA excretion for worker A was highest on the day subsequent to the day with the highest exposure illustrates that MDA may still be excreted after 48 to 72 hours. ${ }^{11}$ Therefore, with dermal exposure to MDA on several subsequent days, the amount of MDA (conjungates) excreted in 24 hour urine samples cannot be clearly appointed to a specific exposure day, indicating the need for a well designed sampling strategy when spot samples are taken. Brumark et al showed a peak of excretion of MDA within 6-11 hours after a 1 hour skin exposure and prolonged excretion after 24 hours. ${ }^{14}$ Boeniger et al indicated that a biphasic pattern of elimination might be expected in cases where exposure will comprise both the inhalation and the skin routes. They suggested that for screening purposes urine samples at the end of shift or at the first void next morning should be collected, in which the next morning elimination might reflect the absorption by skin contact. ${ }^{9}$

In conclusion, both dermal exposure monitoring methods were applicable and showed a compatible performance to assess exposure to MDA in the present exposure scenario where relevant exposure for dermal absorption is mainly restricted to the hands. The concept for a quantitative DOEL seemed to be relevant for MDA and applicable for compliance testing and health surveillance in the situation under investigation, where occupational exposure is dominated by the dermal route.

This study was financially supported by the Dutch Ministry of Social Affairs and Employment. The important contributions of $\mathrm{H}$ van der Waal of Shell, the management of the factory that makes glass fibre reinforced resin pipes, and employees who participated in the study are gratefully acknowledged.

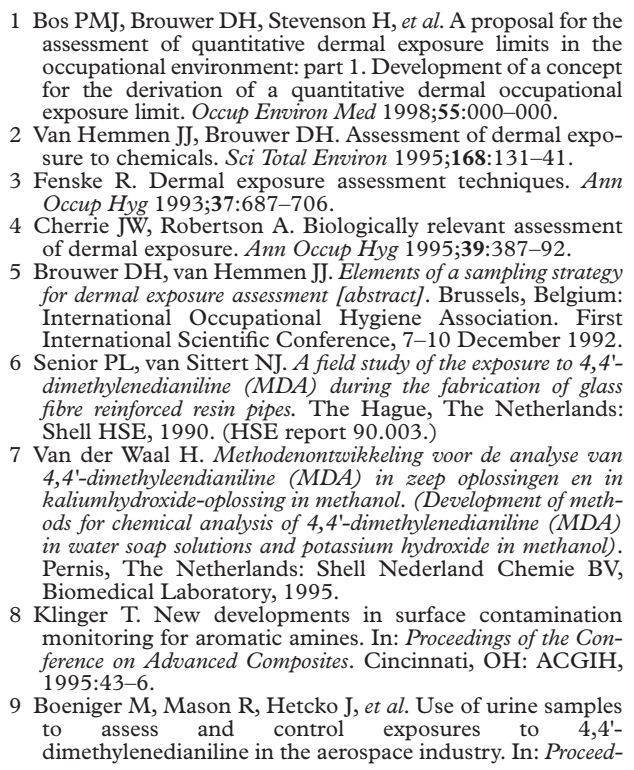


ings of the Conference on Advanced Composites. Cincinnati, OH: ACGIH, 1995:87-111.

10 Cocker J, Gristwood W, Wilson HK. A biological monitoring assessment of exposure to methylene dianiline in manufacturers and users. Occup Environ Med 1994;51 519-22.

11 Boogaard PJ, van der Waal H. Biological monitoring of dermal exposure to 4,4'-dimethylenedianiline (MDA) by determination of $M D A$ in hydrolysed urine. A human volunteer study [internal report]. Rotterdam, The Netherlands: Shell Biomedical Laboratory, 1994.
12 Hornung RW, Reed LD. Estimation of average concentration in the presence of undetectable values. Appl Occup Environ Hyg 1990;5:46-51.

13 Hewitt PG, Hotchkiss SAM, Caldwell J. Decontamination procedures after in vitro topical exposure of human and rat skin to 4,4'-methylenebis[2-chloroaniline] and 4,4'methylenedianiline. Appl Toxicol 1995;26:91-8.

4 Brunmark P, Bruze M, Skerfving S, et al. Biomonotoring of 4,4-methylenedianiline by measurement in hydrolysed urine and plasma after epicutaneous exposure in humans. Int Arch Occup Environ Health 1995;67:95-100.

\section{Occupational and Environmental Medicine - http://www.occenvmed.com}

Visitors to the world wide web can now access Occupational and Environmental Medicine either through the BMJ Publishing Group's home page (http://www.bmjpg.com) or directly by using its individual URL (http://www.occenvmed.com). There they will find the following:

- Current contents list for the journal

- Contents lists of previous issues

- Members of the editorial board

- Subscribers' information

- Instructions for authors

- Details of reprint services.

A hotlink gives access to:

- BMJ Publishing Group home page

- British Medical Association website

- Online books catalogue

- BMJ Publishing Group books.

The web site is at a preliminary stage and there are plans to develop it into a more sophisticated site. Suggestions from visitors about features they would like to see are welcomed. They can be left via the opening page of the BMJ Publishing Group site or, alternatively, via the journal page, through "about this site". 